Institute for Fundamental Theory Preprint UFIFT IFT HEP-91-27

October 1991

\title{
Weight diagram construction of Lax operatiors*
}

DOE/ER/ $40272--140$

DE92 0008781

Steven L. Carbon and Eric J. Piard

Inistitutie for Fundiumental Theory

Depurtimentit of Philsices

University of Flondia, Geimesuldle, FL 39611

Abstruct: We review and expand methods introdinced in our previous paper. It is proved that cyclic: weight diagrams corresponding to representations of affine Lie algebras allow one to construct the associated Lax operator. The resultant Lax operator is in the Minra-like form and generates the modified KdV equations. The algorithm is extended to the supersymmetric case.

* Supported in part by U.S. Department of Energy under contracti No. DE-FG(05-86ER-40272, and a summer grant from the Instititie fior Fundamental Theory.

\section{DISCLAIMER}

This report was prepared as an account of work sponsored by an agency of the United States Government: Neither the United States Governinent nor any agency thereof, nor any of their employees, makes any warranty, express or implied, or assumes any legal liability or responsibility for the accuracy, completeness, or usefulness of any informution, appuratus, product, or process disclosed, or represents that its use would not infringe privately uwned rights. Reference herein to any specific: commercial product, process, or service by trade nume, trademurk, munufacturer, or otherwise does not necessibily constinute or imply its endorsement, recommendation, or favoring by the United States Government or any agency therenf. The views and opinions of authors expressed herein do not necessarily state or roflect those of the United States Government or any agency thereof. 


\section{INTRODUCTION}

Severall approaches tio constructing the Lax operators have been developed. The matrix procedure discussed by Drinfeldi and Sokolov ${ }^{1}$ defines first a matrix eigenvalire equation. The system incorporates knowledge of the Cartan subalgebra and root system of some embedding affine Lie allgebra $\hat{\mathcal{G}}$. Stanting with an affine Iie algebra facilitates the construction of an integrable system from the resulting Lax pair operators. To fix the gauge invariance in the matrix system, the gradation conventions of Drimfeldi and Solkolov require

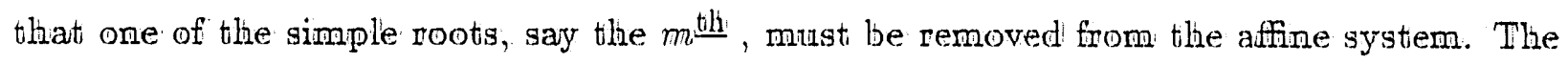
resulting system is denoted by $\left(\hat{\mathcal{G}}, c_{m}\right)$. This is equivalent to deleting the $m$ th Dynkin vertex. For the most part, Drinfeld and Sokolov choose the "canonical" gauge in which to express the coordinate dependent terms. In this gauge, Lax operators generate the regular KdV hierarchyr equations.

If an endpoint vertex is deleted, the remaining matrix system generates a single Lax: operator. For example, Drimfeld and Sokolov find that the canonical representation of $\left(A_{r_{i}}^{(1)}, c_{0}\right)$ creates a Lax operator related to the fundamental representation of $A_{n}$. On the other hand, if the eliminated root splits the Dynkin diagram, a coupled matrix system.

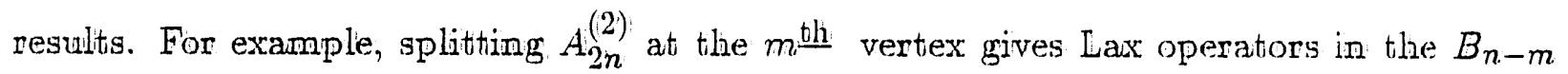
and $C_{m}$ series. Furthermore, the (pseudo)differential operator associated with the $D_{n}$ series is derived using the embedding algebra $D_{n}^{(1)}$.

The modified $\mathrm{KdV}(\mathrm{midV})$, equations can be generated by expresing the coordinate term $q(x)$ in the "diagonal" gauge. The canonical Lax op srators can then be recovered using the well-known Miura transformations. The diagonal gauge is technically simpler than the canonical gauge. Furthermore, the final Lax operator is in a factorized form which has been used to quantize the theory ${ }^{2}$. 
In this paper our focus will be on the explicit construction of Lax. operators in the diagonal gauge as introduced in our previous paper' where we have presented a simple diagrammatic techmique for constructing these (pseudo)differential operators. In most cases; this techmique arrives wt these operators much quicker than a direct application of the scheme of Drinfeld and Solkolov. Furthernore, the scheme also applies to higher representations of the embedding affine Lie algebra. In section II we briefly review affime and non-affime Lie allgebras and construction of weight diagrams. Section III reviews the matrix method of Drimfeld and Solkolov for building Lax operators. Next is a presentation of our method, which replaces the matrix procedure with a scheme utilizimg cyclic weighth diagrams of representations of affine Lie algebras. Section IV presents a proof that the diagrammatic algorithm produces the correct Lax operator. Finally, section $V$ discusses the generalization to Lax operators based on supersyrmmetric affine Lie alkgebras.

\section{REVIEW OF LIE ALGEBRAS AND WEIGHT DIAGRAMS}

As noted in the introduction, each Lax operator can be associated with a representation of some affine Lie algebra. As a result, in this section we give a brief review of relevant aspects from the theory of Lie algebras. In particular we wish to set the notation and discuss the construction of weight diagrams.

\subsection{Basic Lie algebras}

Recall ${ }^{4}$, a basis of generators for a basic semi-simple Lie algebra $\mathcal{G}$ is given by a system 
of roots $E_{\alpha}$, ardid the generattors: $H_{i}$ of itts Cartan sulballgebra 74 . These generators satisfy the commutiation rellations:

$$
\begin{aligned}
& \left.\llbracket H_{i}, H_{j}\right]=0, \\
& {\left[\| H_{i}, E_{\alpha \times}^{\prime}\right]=\alpha_{i}, E_{\alpha}, \quad 1 \leq i \leq r,}
\end{aligned}
$$

where $r$ is the rank of $G$. Each root cam be writtien im the form (alb....). A posittive(negative) root is one whose first non-zero element has a positive(megative) value. There are an equall number of positive and negative roots. A simple positive(megative) root is one which cam not be written as a linear combination of other positive(negative) rootis: with all positive coefficients. We denote the negative simple roots by $E_{i}, 1 \leq i \leq r$, witirch we labell with latin indices: to distimguish them from the full ront system $E_{\alpha}$.

The simple roots, when expressed in the Cartan-Chevalley basis, satisfy the commutation relations

$$
\begin{aligned}
{\left[E_{i}, F_{j}\right] } & =\delta_{i j} H_{i}, \\
{\left[H_{i}, E_{j i}\right] } & =A_{i j} E_{j,}, \\
{\left[H_{i}, F_{j}\right] } & =-A_{i j} F_{j},
\end{aligned}
$$

where $A_{i j}$, for $1 \leq i, j \leq r$, is the Cartan matrix of the Lie algebra.

Below, we will need explicit matrix representations. A straighthforward procedure for computing the entries of the Cartan matrix originates with the Dynkin diagram. Recalli, each of the $r$ vertices of a Dynkin diagram corresponds to a positive simple root vector. Furthermore, the angle between the vectors is indicated by the number of lines connecting their respective Dynkin vertices. The Cartan matrix is then computed by

$$
A_{i j}=\frac{2\left(F_{i}, F_{j}\right)}{\left(F_{j}, F_{j}\right)}
$$

Clearly, the diagonall elements: will all equal two.

Now, one can associatte uniquely tio every irreducible representation of a basic Lie algebra a highest weight vector, that is a certain linear combination of positive simple rootis 
which hass: the propertiy of beimg anmilhillated by some $E_{\alpha}$ for appropriate $\alpha$. Comverselyr, tio every limear combination of positive simple roots there corresponds a highthest weight vector of some indeducible representiation. Furthermore, for each highthest weight one can construct a weightht diagram which encodes all rellevant information concerning the particular representabtion studlied, e.g.., from it one can build expllicit mattrix representations of the generattors of the Cartam suballgebra: as well as the various raising and lowerimg opuratons. The levell of a weighth is the mumiber of lowerimg operattors applied tio the highthest weight which produces that weighth. Finally, the height $\gamma$ of the weight diagram is the levell of the lowrest weight.

Weight diagrams are generated by sulbtracting rows of the Cartan matrix inditially from the highthest weight vector. Rulles of construction can be summed up as follows:

1. Subtoract the $i$ th row of the Cartan matrix mimes from a weighth vector whose $i$ thi component has a positive vallue $m$.

2. When weight vectors have more than one positive component, subtract all possible permutations of the appropriatte Cartan rows.

A theorem due to Dymkin 5 stattes that the finall weighth diagram is always "spindle shaped". In other words: i) the number of weight vectiors at the levell $k$ is equali to the mumber at level $\gamma-k$, ii) the number of weights at level $k+1$ is greater than or equal to the number at level $k$ for $k<\frac{7}{2}$.

For an explicit example consider the algebra $A_{2}$. Though this is almost a trivial case, the results will be useful for the next section. The Dynukin diagram is given by

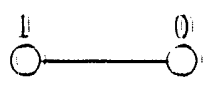

where, recall, the single bar represents $120^{\circ}$. The Cartan matrix is then easily found to be

$$
A=\left(\begin{array}{cc}
2 & -1 \\
-1 & 2
\end{array}\right)
$$


The highthest weighth vection of the fundamentall representattion is (10) ${ }^{6}$. Since a posittive one appears in the first place, we stulbtract the flinst row of the Carthan mattrix one thime. Thiss. gives: the weightht (-11). Now, dhe to the one in the second place, we sulbtract the second row of the Cartan matrix once to get $(0-1)$. Thiss complettes the process since no positive componentts nemaim. The resullt is the theightht two weight dliagram

$$
\begin{gathered}
(101))_{1} \\
\Downarrow \mathbb{1} \\
(-11)_{2} \\
\downarrow \mathbb{1} \\
((0)-11)_{3}
\end{gathered}
$$

where the sulbscripts on the weight vectors indicatte a coumiting of the vections. The ones. adjiacent to the arrows represent the normalization factions of the corresponding negative simple roots. These values are fixed by the commutation relations (2.1.2). To simplify our dliagrams, we will not display valies: of unity. Latter, we will see that the procedure for buillding weight diagrams is slighthly modiffed in the supersymmetric case.

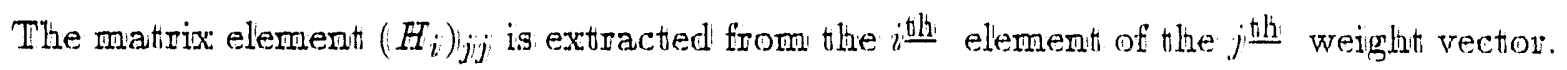
Setting the off-diagonall elementis to zero gives

$$
H_{1}=\left(\begin{array}{ccc}
1 & 0 & 0 \\
0 & -1 & 0 \\
0 & 0 & 0
\end{array}\right) \text {, }
$$

and

$$
H_{2}=\left(\begin{array}{ccc}
0 & 0 & 0 \\
0 & 1 & 0 \\
0 & 0 & -1
\end{array}\right) .
$$

The mattrix entry of the negative simple root $\left(E_{i}^{\prime}\right)_{j k}$ is assigned its normallization factor if the $k$ the weight vector brauches intto the $j$ the weight vectior as a restull of sulbtracting the $i$ thew of the Cartan matrix in the process. Other entries are by defatult zero giving the mattrices

$$
E_{u}=\left(\begin{array}{lll}
0 & 0 & 0 \\
1 & 0 & 0 \\
0 & 0 & 0
\end{array}\right),
$$

and 


$$
E_{2}=\left(\begin{array}{lll}
0 & 0 & 0 \\
0 & 0 & 0 \\
0 & 1 & 0
\end{array}\right)
$$

\subsection{Affine Lie algebras}

An affine Lie allgebra is defined to be a tensor product of the basic Lie allgelbra witth a Laurent pollymomiall allgetbra $C\left(\left(\lambda, \lambda^{-1}\right)^{7}\right.$. The simple root systtem of an affine Lie allgebra comisistis: of the simple roots of the correspondlimg, Lie allgebra, and an affine root withich projects: ontto the basic Lie allgebra as the corresponding, lowest rooth. IIm the CarttanChevalley basis the simple roots of an affine lie allgebra obey the commuthation relations.

$$
\begin{aligned}
& \left\|H_{i}, H_{j i}\right\|=0, \quad \quad\left[E_{i}, F_{j i} \rrbracket=\delta_{i j, j} H_{i},\right. \\
& \left\|H_{i}, E_{j j}\right\|=A_{i j} E_{j i}, \quad \llbracket H_{i}, F_{j j} \|=-A_{i j j} F_{j},
\end{aligned}
$$

where now (1) $\leq i, j \leq \leq r$. Unllike simple root systlems of nom-affine Lie allyebras, there existis a limear relattion among the positive(negative)) simple rooths in affine allgebras. Consequentlly, affime Lie allgebras are imflinitte dimensionall.

Affime Lie allgebrass are invariant under an outter automorphism $\tau$. The ordler $k$ of the autiomorpthism $T$ is the smallest integer such that $\pi^{k}=1$. A twisted affine algetbra is one swch thath $k \geq 2$. Let 1 us denote a generall affine Lie allgebra $\hat{\mathcal{G}}^{\left(k_{i}\right)}$. Notte, there may exists move thithan one affime extension of a partictular basic Lie allgebra, e.g., An: extends to both the affine allgebra $A_{n}^{(1)}$ and the twistied affine aulgebra $A_{n}^{(2)}$.

In generall, dhe to the linear combination among simple roots, weighth diagrams of aiffine representations have infinitte extenth. However, sc me affine representations give cyclic weight diagrams of fimitie extenth. In fact, it is these cyclic cases thath are cruciall to the scheme below. To produce a cyclic weighth diagram, the affine component which is appended to the highthest weightit vectior of the underlying non-affine Lie allgebra, unlike the nor-auffine weight compoments, may have to be assigned a megattive vallue.

As an explicit example, consider the non-twisted affine allgebra $4_{2}^{(1) !}$. To generaite the cyclic weight diagram correaponding to the canonical representation; start with the 
weightht, vecttor ( $-11(0))$, where - 1 correspondls to the affine roott. Figure 11 gives the Dyrnkin diagram and sulbsequent Cartlan mattrix which then generates the dlisplayed restulltant weighth diliagrarm.

Thiss particullar cyclic weighth dliagram can furtther be thoughth of ass the affine extlension of the hinghesth weight diagram based on the fiumdamemtall representation of the basic Lie allgebra $A_{2}$. Thuis: is: easiy to see by removing everyw here the componemt dute to the affine root. However, thiss is not allways the case. For example, Figure 2 ellisplays the cyclic weightht diagram constructed with the weighth vectior $(-211)$, where now the affine component is -2. Alththough (11) generattes: the highthest weighth diaugram of the adjoimt representation of .42 , we see by comparing witth Figure 3 thath the affine extension conttaing an extra zero. weightht ((000)).

In generall, the affine component in the affinely extlendid vector, associalted with the highthest weighth vectior of a basic Lie allgebra, willl allways be negattive. However, we will give an example below shewing that some supersymmetric cases require positive affine componentis.

\section{LAX OPERATOR CONITRUCTION}

\subsection{Standard construction of Lax operators}

The Lax operattor ${ }^{8} L(x, t)$ is deffined to be linear and Hermitian. Furthermore, it is required to have the propertiy that its eigenvalues are constiant under nonlinear evolution. In otther words, for constant eigenvalues $\mu$ the eigenfunctions of the scallar characteristic 
equaltiom

$$
L((x, t)) \phi\left(\left(x, f^{\prime}\right)\right)=\mu \phi((x, t))
$$

willl evolve nomllinearly. The eigenfunctions thave impoed wpom them the propertiy they are unittary witth respect to the origin, ine.,

$$
\phi\left(\left(x, t^{\prime}\right)\right)=U(t) i \phi((x,(0)) .
$$

Them, thiss: nomllinear bethavior will be governed by some operattor $A(t)$ ), which may be momlinear, vina the equattion

$$
\left.\left.\frac{\partial \phi(x, t))}{\partial t}=\frac{\partial U((t))}{\partial t} \phi(x,(0))=A(t)\right) \phi(x, t)\right),
$$

where we have dlefimed

$$
\frac{\partial U(t))}{\partial t} \equiv A(t) U(t i)
$$

The pair of operattors $L(x, t)$ and $A(t)$ is called the Lax pair.

Because $L(x, t)$ is a Hermitiam operator itts dynamics is gemeratted by the unitary op. erattor, ii.e.,

$$
\left.U^{\dagger}((t)) L((x, t)) U(t)\right)=L((x, 0))
$$

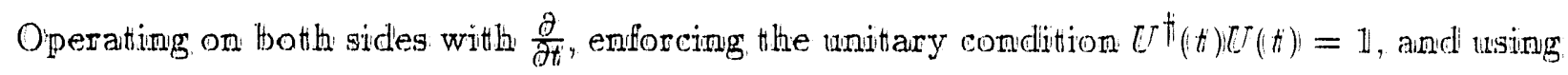
(3.1.4) gives

$$
U^{\dagger}((t))\left(\frac{\partial L(x, t))}{\partial t}-\llbracket A(t), L(x, t) \rrbracket\right) U(t) i=0
$$

The final result is the differentiall scallar Lax equation

$$
\frac{\partial L(x, t)}{\partial t}=\|A(t), L(x, t)\|
$$

which generates the imtegrable KdV equations. Physically, the $\mathbb{R} d V$ equations describe the governing motion of solitons. Further, the spectrall parametier $\mu$ is proportionall to the speed of propagation of the solition wave. This makes sense since sollitons are non-dlispersive and $\mu$ is required to be independent of time. 
The mattrix construction of Lax operattors utillizing generattors of some embedlding affine Lie allgebra $\hat{\mathcal{G}}^{\left({ }^{(k))} \text {, reviewed by Drimfield and Solkolov }\right.}{ }^{\sharp}$, beginus: with a mattrix operattor of the form

$$
\mathcal{L}=I \frac{\partial}{\partial x}+\Lambda+q(x)
$$

where I dlenottes, the $N \times N$ dimensionall unitt mattrix, and tho simpllify nottation we thave suppressed the argument th. The third therm is discussed below. The second therm is generaited by the negative simplle roots: of the embedlding affine Lie allgebra. In the graidation convemtiong: of Drinfeld and Solkolow, we thave the circulamt. maitrix

$$
\Lambda=\sum_{i=01}^{r} c_{i} E_{i} .
$$

The procedure is them to reduce the system of linear equattions given by the kernel mattrix equation

$$
\mathcal{L} \cdot \vec{\psi} \cdot(x)=0
$$

where $\vec{\psi}=\left(\psi \psi^{\prime}, \psi_{2}, \ldots, \psi, w\right)$, to the linear differential eigemvallue equation $(3.1 .1)$, where the varum solution $\phi$ is a frunction of the components of the eigenfunction $\vec{p}$. Drinfeld and Sokolov show that such reduction is possible if one removes a simple root, say the $m$ 望 , from the affine root system. They denote this sittuation $\left(\hat{\mathcal{G}}^{\left(k^{\prime}\right)}, c_{m}\right)$, which is in the homogeneouss or standard gradation ${ }^{9}$. The coefficients in (3.1.9) are then aissigned the vallues $c_{i \neq m}=1$ and $c_{m}=\lambda$, where $\lambda$ is a constanit function of the spectrall parameter $\mu$.

Removing an element from the simple root system is equivallent to dielleting thitie corresponding vertex from the Dynkin diangram. This, when an extremall vertex is deletted, the system $\left(\hat{\mathcal{G}}^{\left(k^{2}\right)}, c_{m}\right)$ represents: a singlle residual basic Lie allgebra. Removing the affine vertiex obviously gives: $\mathcal{G}$. On the other hand, deleting intternal vertices splits the Dynkin diagram intio two sections, corresponding to a pair of basic Lie allgebras. In botth situattions, a pair of (pseudb) differentiall operattors is found whose product gives the Lax cperattor $L$ of $\mathrm{Eq}$ q. (2.11.7)). 
To dletermine the exact form of the vacuum solution $\phi$ recall that negative simple roots are lowering operattors on systhem eigensthattes: Furtither, removal of a root in the affine systtem produces the simplle root systtem of a non-affine Lie allgebra. Thus, dive to the linear combination among the roots: of the affine systtem, one root must be sirngled out to act ass a conventionall sttarte raising operattor. This rolle is given to the removed roott. Thus, the vacuum eigensithatte will be ammithillatedl by a vacum projection operattor $\Delta^{-}$defined by

$$
\Lambda=\sum_{i=0}^{n} E_{i \neq m i}+\lambda E_{m i} \equiv \Delta^{-}+\lambda E_{m i} .
$$

This requirement fixes the scallar vacuum solution $\phi$ by seitimg it equal to a linear combiwattion of the components of $\vec{\psi}$ surch thait

$$
\Delta-\vec{k}=0
$$

is satisffied. A direct relation between the scallar operattor $L$ and the mathix operattor $\mathcal{L}$ will be given in the rext section.

For the kermel equation $(3.1 .10)$ ) to produce a unique solution, we require that the number of independent degrees of freedom equal the rank of the embedding affine Lie allgebra $\hat{\mathcal{G}}^{(k)}$, or equivallentlly the residuall systhem $\left(\hat{\mathcal{G}}^{(k)}, c_{m}\right)$. The extra degrees of freedom generatte gauge invariance. To fix the ganuge invariance, one must fund a matrix operaitor $S(x)$ that enforces the gauge transtormation

$$
\mathcal{L}_{0}=e^{a d} S \dot{L}
$$

where ad denotes the adjoint mapping. The gauge freedom present in Eq. (3.1.10) alllowls one freedom in determining the form of the coordinate dependent term $q_{0}(x)$, i. e.,

$$
\mathcal{L}_{0}=I \frac{\partial}{\partial x}+\Lambda+q_{0}(x)
$$

Drimfeld and Sokolov find the sufficient condition that $S \in C^{\infty}(\mathrm{R}, \eta)$, where $\eta$ is generated by the positive simple roots $F_{i}, i \neq m$. 
Many authors, including Drinfeld and Sokolov, work most freq'ently in the "canonicall" gauge. However, in this paper we choose to work in their "dliagonal" gauge which has the form

$$
q^{\operatorname{diag}}(x)=\left(\begin{array}{ccccc}
q_{1} & 0 & \ldots & 0 & 0 \\
0 & q_{3} & \ldots & 0 & 0 \\
\vdots & \vdots & \ddots & \vdots & \vdots \\
0 & 0 & \ldots & q_{N-1} & 0 \\
0 & 0 & \ldots & 0 & q_{N}
\end{array}\right)
$$

Thiss gauge leards to the convenient form

$$
q^{d i a g}=\sum_{i=0}^{r} v_{i}\left(q_{1}, q_{2}, \ldots, q_{N}\right) H_{i}
$$

which is in the canonicall or principal gradation ${ }^{9}$. Here, the functions $v_{i}$ are linear combimations of the elements $q_{i}$. In this gauge, the gauge term $q^{\text {diag }}$ aissociated with $\left(\hat{\mathcal{G}}^{(k)}, c_{m}\right)$. is the special case where the sum excludes $i=m$. The Lax operation $L^{\text {diag }}$ generattes the mKdV equations, and is relatted to $L^{c a n}$ via the well-known Miura transformations.

\subsection{Diagrammatic construction of Lax operators}

To exploit gauge invariance of the Lax operators, one should choose a $q(x)$ gauge most suited to ones needs. Here, we are interested in developing a diagrammatic scheme for constructing $L$. In this regard, the diagonal gauge proves more useful than the other choices. In this section, we will demonstrate how the diagonal gauge allows one to build Lax operatiors directly from cyclic weight diagrams of representations of affine Lie algebras.

To motivate the algorithm, we first review the construction of $L$ by solving the matrix system $\mathcal{L} \vec{\psi}=0$. For the present discussion, it will be sufficient to consider embedding algebras of the form $\left(\hat{\mathcal{G}}^{(k)}, c_{0}\right)$ where the affine vertex is deleted. Thus, the diagonal gauge 
simply reduces to the form

$$
q^{\operatorname{diag}_{(}(x)}=\sum_{i=1}^{r} v_{i}(\mathrm{i} x) \mathrm{H}_{i},
$$

where we have excluded $H_{0}$, from the sum.

Consider again the canonical representation of the embediding affine Lie algebra $\left(A_{2}^{(1)}, c_{0}\right)$ presented iru Figure 1. Matrix representations of the Cartan matrix can be read-off from the cyclic weight diagdam, producing the previous results given in Eqs. (2.1.6) and (2.1.7). Similarly, the matrix representations of the simple noots are easily found to give

$$
\Lambda=\left(\begin{array}{lll}
0 & 0 & \lambda \\
1 & 0 & 0 \\
0 & 1 & 0
\end{array}\right)
$$

where the effect of the affine root, indicated in Figure 1 by the dashed arrow line, is assigned the value $\lambda$. Plugging these values into the kernel equation (3.1.10) produces the system: of equations

$$
\begin{aligned}
{\left[\partial+v_{1}\right] \psi_{1} } & =-\lambda \psi_{3}, \\
{\left[\partial-v_{1}+v_{2}\right] \psi_{2} } & =-\psi_{1}, \\
{\left[\partial-v_{2}\right] \psi_{3} } & =-\psi_{2} .
\end{aligned}
$$

Here, on the right-hand side we have placed the terms due to the matrix $\Lambda$.

The vacuum condition (3.1.12) determines the scalar function to be $\phi=\psi_{3}$. Thus, we must solve by starting with the last equation. First, we multiply this equation through by $\left[\partial-v_{1}+v_{2}\right]$, and then eliminate $\psi_{2}$ using the second equation. This gives

$$
\left[\partial-v_{1}+v_{2}\right]\left[\partial-v_{2}\right] \psi_{3}=\psi_{i}
$$

Next, multiply through by $\left[\partial+v_{1}\right]$ and use the top equation in (3.2.3). The result, is the scalar Lax eigenvalue equation

$$
\begin{aligned}
L^{\left(A_{2}^{(1)}, \infty_{0}\right)} \psi_{3} & =\left[\partial+v_{1}\right]\left[\partial-v_{1}+v_{2}\right]\left[\partial-v_{2}\right] \psi_{3} \\
& =-\lambda \psi_{3},
\end{aligned}
$$

where the spectral parameter is given by $\mu=-\lambda$. Imposing the field redefinitions

$$
q_{1}=v_{1}, \quad q_{2}=v_{2}-v_{1}
$$


gives the standland form

$$
L^{\left(A_{2}^{(1)}, \sigma_{0}\right)}=\left[\partial+q_{1}\right]\left[\partial+q_{2}\right]\left[\partial-q_{1}-q_{2}\right]
$$

This example exhibits a common feature relevant for our scheme below. When the vacuum condition (3.1.12) requires the scalar eigenfunction to be given by a single component of the eigenfunction, say $\phi=\psi_{i}$, then the resulting charactenistic equation satisfies

$$
L \psi_{i}=\mu \psi_{i}
$$

Consequently, the system reduction must start with the $i \frac{\text { th }}{2}$ equation in the matrix system, and proceed upward till the top equation is reached. If $i \leq N$ the process continues with the bottom equation and moves upward until the $i$ th equation is reached again. We shall refer to this case as trivial since the corresponding cyclic affine weight diagram is linear, containing no branch points. A second feature brought out in this example, is that the number of factors in the resultant Lax operator (3.2.7) is equal to the number of weights in the weight diagram. Unfortunately, this is valid only for trivial cases. Nevertheless, this last observation is key to our scheme.

To highlight one more property of the general procedure we turn to a non-trivial example. For this, we require a representation of an affine Lie algebra whose cyclic weight, diagram has at least one branching point. Thus, consider the canonical representation of the affine algebra $\left(D_{4}^{(1)}, c_{0}\right)$. Figure 4 presents the Dynkin diagram, Cartian matrix and corresponding cyclic weight diagram which has two branch points. Reading off from the weight diagram gives

$$
\Lambda=\left(\begin{array}{llllllll}
0 & 0 & 0 & 0 & 0 & 0 & \lambda & 0 \\
1 & 0 & 0 & 0 & 0 & 0 & 0 & \lambda \\
0 & 1 & 0 & 0 & 0 & 0 & 0 & 0 \\
0 & 0 & 1 & 0 & 0 & 0 & 0 & 0 \\
0 & 0 & 1 & 0 & 0 & 0 & 0 & 0 \\
0 & 0 & 0 & 1 & 1 & 0 & 0 & 0 \\
0 & 0 & 0 & 0 & 0 & 1 & 0 & 0 \\
0 & 0 & 0 & 0 & 0 & 0 & 1 & 0
\end{array}\right)
$$


The branch points have manifested themselves by placing more than one non-zero entry in the second and sixth nows. Now, further reading off the elements of the Cartan matrices: gives the system of equations

$$
\begin{aligned}
& {\left[\partial+v_{1}\right] \mid \psi_{1}=-\lambda \psi_{\eta},} \\
& {\left[\partial-v_{1}+v_{2}\right] \psi_{2}=-\psi_{1}-\lambda \psi_{8},} \\
& {\left[\partial-v_{2}+v_{3}+v_{4}\right] \psi_{3}=-\psi_{3},} \\
& {\left[\partial-v_{3}+v_{4}\right] \psi_{4}=-\psi_{3},} \\
& {\left[\partial+v_{3}-v_{4}\right] \psi_{5}=-\psi_{3},} \\
& {\left[\partial+v_{2}-v_{3}-v_{4}\right] \psi_{b_{3}}=-\psi_{4}-\psi_{5},} \\
& {\left[\partial+v_{1}-v_{2}\right] \psi_{7}=-\psi_{6},} \\
& {\left[\partial-v_{1}\right] \psi_{8}=-\psi_{i} \text {. }}
\end{aligned}
$$

The vacuum condition (3.1.12) produces two distinct solutions, $\psi_{s}$ and the linear combination $\psi_{4}-\psi_{5}$. Here, we consider the first case. Proceeding as before, we eliminate $\psi_{n}$ and $\psi_{7}$ in the last two equations to get

$$
\left[\partial+v_{2}-v_{3}-v_{4}\right]\left[\partial+v_{1}-v_{2}\right]\left[\partial-v_{1}\right] \psi_{38}=-\psi_{4}-\psi_{5}
$$

Now, we encounter a well-known technical problem not found in the trivial case. The components $\psi_{4}$ and $\psi_{5}$ can not both be simultaneously eliminated since the expressions $\left[\partial-v_{3}+v_{4}\right]$ and $\left[\partial+v_{3}-v_{4}\right]$ do not commute. This dilemma is directly linked to the fact the corresponding cyclic weight diagram has a branch point connecting the fourth and fiftih weights to a single weight located below them.

To overcome this obstacle the pseudo-differential operator $\partial_{x}^{-1}$ must be introduced. Its operation on any function $f(x)$ is given by the expansion

$$
\partial_{x}^{-1} f(x)=\sum_{i=0}^{\infty}(-1)^{i} f^{(i)}(x) \partial_{x}^{-1-i}
$$


Utilizing the preudio-differentiall operator, we rewritie the fourth equation in $(3: 2.10)$ ) as

$$
\left.\psi_{4}=-\left[0-v_{3}+v_{4}\right]\right]^{-1} \psi_{3} .
$$

Thus, the combined effect of the bottom five equations is.

$$
\begin{aligned}
\psi_{3}= & \left.\left\{\left[\partial-v_{3}+v_{41}\right]^{-1}+\left[\partial+v_{3}-v_{4}\right]\right]^{-1}\right\}_{1}^{-1}\left[\partial+v_{2}-v_{31}-v_{4}\right] \mid \\
& \times\left[\partial+v_{1}-v_{2}\right]\left[\partial-v_{11}\right] \psi_{3} .
\end{aligned}
$$

A thelpfinll identity we use repeatedlly is

$$
\left\{A^{-1}+B^{-1}\right\}^{-1}=\left\{A^{-1}[A+B] B^{-1}\right\}^{-1}=B[A+B]^{-1} A
$$

When applied to Eq.(3:2.14), a cancellation occurs among the $v_{i}$ 's appearing in the curly brackets. This simplifies the expression to

$$
\psi_{3}=\frac{1}{2}\left[\partial-v_{3}+v_{4}\right] \partial^{-1}\left[\partial+v_{3}-v_{4}\right]\left[\partial+v_{2}-v_{3}-v_{4}\right]\left[\partial+v_{1}-v_{2}\right]\left[\partial-v_{1}\right] \psi_{4} .
$$

Continuing, incorporating the next two equations in (3.2.10) requires a second application of the relation (3.2.15): Finally, the Lax operation based on $\left(D_{4}^{(1)}, c_{(0)}\right)$ with vacutum $\psi_{\mathrm{s}}$ is

$$
\begin{aligned}
L= & \frac{1}{4} \partial^{-1}\left[\partial+v_{1}\right]\left[\partial-v_{1}+v_{2}\right]\left[\partial-v_{2}+v_{3}+v_{4}\right]\left[\partial-v_{3}+v_{4}\right] \\
& \times \partial^{-1}\left[\partial+v_{3}-v_{4}\right]\left[\partial+v_{2}-v_{3}-v_{4}\right]\left[\partial+v_{1}-v_{2}\right]\left[\partial-v_{1}\right]
\end{aligned}
$$

Using the field redefinitions

$$
\begin{gathered}
q_{1}=v_{1}, \\
q_{3}=-v_{2}+v_{3}+v_{4}, \quad v_{4}=-v_{1} \\
q_{3}+v_{4}
\end{gathered}
$$

we get

$$
L=\frac{1}{4} \partial^{-1}\left[\partial+q_{1}\right]\left[\partial+q_{2}\right]\left[\partial+q_{3}\right]\left[\partial+q_{4}\right] \partial^{-1}\left[\partial-q_{4}\right]\left[\partial-q_{3}\right]\left[\partial-q_{2}\right]\left[\partial-q_{1}\right]
$$

which is proportional to the standard result.

We have chosen these two examples because they introduce the techniques needed to generate Lax operators associated with even the most complicated algebraic systems. 
Furthermore, they show how closely the structiune of cyclic affime weighth dliagrams is liniked with the comstruction of generall Lax operators. As a result, we propose a set of foum steps. which allows: one to construct Lax operations associated with cyclic representhations of arfime Lie allgetbras.

First, we propose that to every weight vector of an affine cyclic weight diagram one can associate an operation as follows:

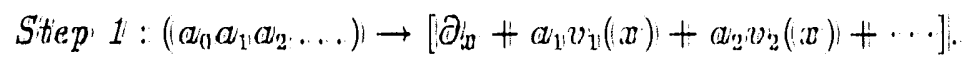

The coefficient $a_{0}$ does not appear on the right-hand side as it corresponds to the deleted vertex. Now, even before we specify how to build $L$ from these factors, a signifficant conchusion can be inferned from this rule. Namely, in the context of scalle invariant theories, two Lax operators $L$ and $L^{\prime \prime}$ will be equivalent if their respective cyclic weighth diagrams have the same shape, normalization factors and number of weight vertors, and if there exists an isomorphism between the coefficients of the weight factons. In facti, some cases relax the condition that the number of weights in the two systems be equal.

Second, we introdice a step which is designed to facilitate the construction of Lax: operators when branch points exist in the corresponding weight diagram. Essentiallily, this step reduces more complicated non-trivial cases to a sum of manageable triviall cases by reducing the branched weight diagram to a sum a linear subdiangrams.

Stiep 2:: Replace branching weight diagmamis. by the y:umi of linear subdzagmamis, each represenitinig a verticicul roulte beginning! writh, and ending on, the vac'unm, weight(y).

For example, Figure 5 presents the four linear subgraplis associatted with the canonical representation of $D_{4}^{(11) !}$.

In drawing cyclic weighth dias ams, it is important that the arrows generatted byr the deleted vertiex are distinguished from the others. Our convention is to use dashed lines. 
Fiurtihermore, the dimection of the annows musti also be notted. The Lax operattors associatted with each subdiangram are then constructed as follows:

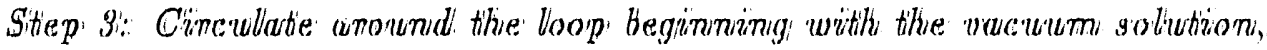

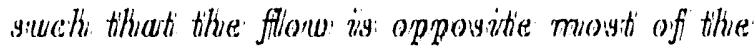

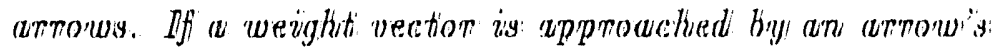

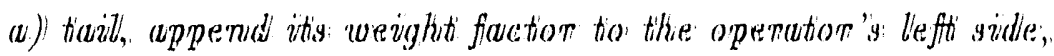

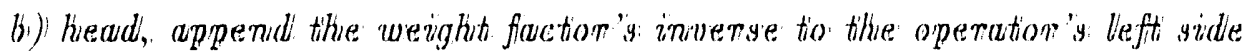

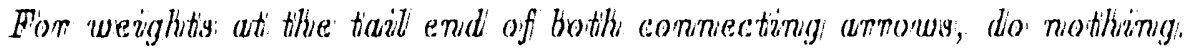

Mrultiply by the prodiuct of the conrespondinug normalization ficuctions.

The loop is to be circullated in a direction opposite most of the arows so thitat the leading therm of the Lax operatior $L=\partial^{n}+\ldots$ has: positive exponent, i. e.., $m_{i}>0$.

For triviall cases, this completes the computation of $L$. However, for mon-triviall cases with branching weight dliagrams: we can not naively build the final Lax operator from a sum of its constituent limear subgraphs. Instread, as we shalli prove in the nexth section, they are added together analogously to how one comptites totall resistance of resistors in paraillell.

Sitep 4: The Lax operution iy given by the inverye of the sumi of

the inveryes, of the operutiony reysulting from step three.

For example, in the non-triviall case $\left(D_{4}^{(11))}, c_{0}\right)$, with $\phi=\psi$ s as before, we build four operators corresponding to the linear sulbdiagrams in Figure 5,

$$
\begin{aligned}
L_{1}= & {\left[\partial-v_{1}\right]^{-1}\left[\partial+v_{1}\right]\left[[ \partial - v _ { 1 } + v _ { 2 } ] \left[[ \partial - v _ { 2 } + v _ { 3 } + v _ { 4 } ] \left[\left[\partial-v_{3}+v_{1}\right]\right.\right.\right.} \\
& \times\left[\partial+v_{2}-v_{3}-v_{4}\right]\left[\partial+v_{1}-v_{2}\right]\left[\partial-v_{1}\right], \\
L_{2}= & {\left.\left.\left.\left[\partial-v_{1}\right]^{-1}\left[\partial+v_{1}\right]\left[\partial-v_{1}+v_{2}\right]\right]\left[\partial-v_{2}+v_{3}+v_{4}\right]\right]\left[\partial+v_{3}-v_{4}\right]\right] } \\
& \left.\times\left[\partial+v_{2}-v_{1}-v_{4}\right]\right]\left[\partial+v_{1}-v_{2}\right]\left[\left[\partial-v_{1}\right],\right. \\
L_{3}= & {\left[\partial-v_{1}+v_{2}\right]\left[\left[\partial-v_{2}+v_{3}+v_{4}\right]\left[\left[\partial-v_{3}+v_{4}\right]\right]\left[\partial+v_{2}-v_{3}-v_{3}\right]\right.} \\
& \times\left[\partial+v_{1}-v_{2}\right]\left[\partial-v_{1}\right],
\end{aligned}
$$


andil

$$
\begin{aligned}
L_{4}= & {\left.\left.\left.\left.\left[\partial-v_{11}+v_{3}\right] \| \partial-v_{2}+v_{3}+v_{4}\right]\right]\left[\partial+v_{3}-v_{4}\right] \| \partial+v_{2}-v_{3}-v_{3}\right]\right] } \\
& \left.\left.\times\left[\partial+v_{1}-v_{2}\right]\right]\left[\partial-v_{11}\right]\right] .
\end{aligned}
$$

Factoring ow common therms, we find

$$
\begin{aligned}
& L^{-1}=\sum_{i=1}^{4}\left(\left(L_{i}\right)\right)^{-1} \\
& \left.\left.=\left[\partial-v_{1}\right]\right]^{-1}\left[\partial+v_{1}-v_{2}\right]\right]\left.^{-1}\left[\partial+v_{2}-v_{3}-v_{4}\right]\right|^{-1} \\
& \left.\left.\left.\times\left\{\left[\partial-v_{3}+v_{4}\right]\right]^{-1}+\left[\partial+v_{3}-v_{4}\right]\right]^{-1}\right]_{1}\left[\partial-v_{2}+v_{3}+v_{4}\right]\right]^{-1} \\
& \left.\left.\left.\times\left[\partial-v_{1}+v_{i_{2}}\right]\right]^{-1}\left[\partial+v_{1}\right]\right]^{-1}\left\{[]+\left[\partial-v_{11}\right]\right]^{-1}\left[\partial+v_{1}\right]\right\} .
\end{aligned}
$$

By traking the reciprocall, and simplifying, we reprodice the previous result Equ(13.2.17).

To end this section we consider the alltermattive vacutum choice $\psi$ - $\psi$.4. It should be o. vious that since we are dealling with cyclic weighth diagrams, Lax operattors associatted wititi other vacuum stlattes: can be achieved by cyclically permutting factors in the primary Lax. operatior. Thus, this second vacuum choice immediately gives the Lax operator

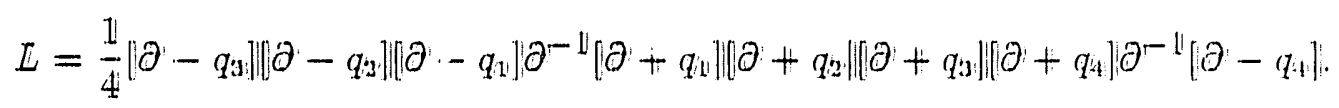

\section{PROOF OF DIAGRAMMATIC SCHEME.}

To prove the equivalence between the matrix systlem $\mathcal{L}_{\vec{\psi}} \vec{p}=0$ and the diagrammatic allgorittim, we begin by rewriting the former as

$$
\vec{D} \cdot \vec{\psi}(x)=-\Lambda \cdot \vec{\psi}(x)
$$

where, to simplify notiation, we have defined

$$
\mathcal{D} \equiv \mathcal{L}-\Lambda=I \frac{\partial}{\partial x}+q(x) .
$$


The stibuctume of the associatted cyclic weighth diagram is encodled entimelly im the maattrix $\Lambda$. Sipecifficallly, recalll that the generall mattrix element $\Lambda_{i j}$ is proportionall tio $\lambda$ it the dirfference bettweem the $i$ the weighth and the connectlimg, jith weighth equalis: the elimimatted soot of the

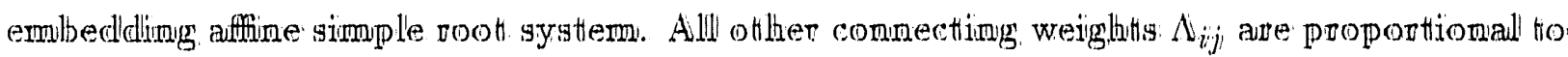
1. Oitherwise, the mattrix: element is assiggned the vallue (0). In alll cases: the proportionallitiy comstrant is the mormallization factor of the commectimg rooth.

We construct the proof in stayges. For the first stange we consicler the triviall case, i.e., a

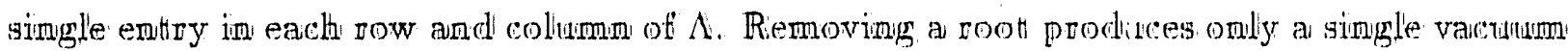
statte. This stage corresponds to weightht diagrams with mo branch poimts and only one arrow associatted with the elliminatted root. Clearly, we cam rearoange the matrix equations in $L \vec{L} \vec{p}=(0)$ such than the vacturm state equation appears lasth. Furthermore, it cam be

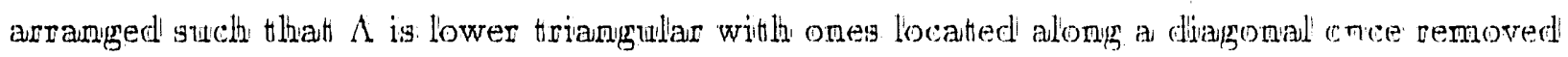
form the main diagonall, except for the eliminated root whose coefficient $\lambda$ appears in the upper righth-hrand cormex, i. e., $\Lambda$ is a circullant matrix. Thus, the Lax eiggenvallue equation becomes

$$
L_{\psi} \psi_{N}(x)=H_{H} \psi_{N}(x)
$$

Since $\mathcal{D}$ is diagonall, the $k$ th

$$
\mathcal{D}_{k i} \psi_{k}=-\sum_{i=1}^{N} \Lambda_{k i} \psi_{i}, \quad k_{i}=2,3, \ldots, N
$$

Clearly, simce $\Lambda$ is a circullant mattrix as specifled above, the inequalityr $i<k$ holds for $k \neq 1$. Next, by repeatedlly replacing the finction $\psi_{i}$, appearing on the rightho-thand sidle, 


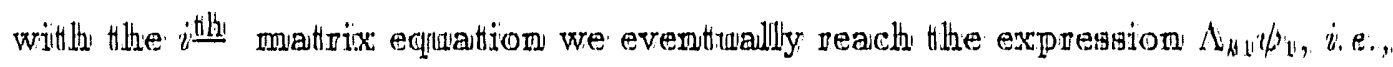

$$
\begin{aligned}
& \mathcal{D}_{N: \psi_{N}}=-\sum_{i=1}^{N} \Lambda_{N i} \psi_{i} \\
& =\sum_{i=1}^{N} \sum_{j=1}^{N} \Lambda_{N i} \mathcal{D}_{i}^{-1} \Lambda_{i j i} \psi_{j j}
\end{aligned}
$$

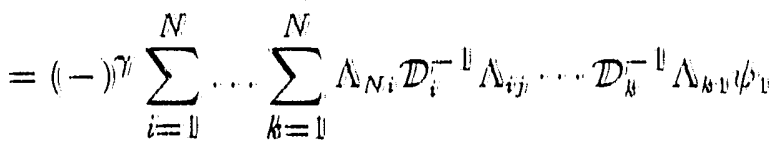

where $\gamma$ is the theighth of the cyclic weighth dliagram. Due to the stuccessive appllications: of the statte lowerimg operattors $\Lambda_{i j}$ with $l i>j$, this equattion is intterpretted as thalking the hinghest sthate $\psi$, and lowering it to the vacutum statte $\psi^{\prime}$.

Replarcings $\psi$, thitought

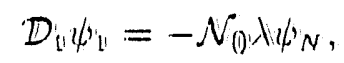

where we have used $\Lambda_{I N}=N_{\text {(n) }}$ which excites the level of the stathe since $1<N$, gives

$$
\mathcal{D}_{N} \psi_{N}=-(-)^{\gamma} \mathcal{N}_{(0) \lambda} \sum_{i=1}^{N} \ldots \sum_{k=1}^{N} \Lambda_{N i} \mathcal{D}_{i}^{-1} \Lambda_{i j} \cdots \mathcal{D}_{k}^{-1} \Lambda_{k 11} \mathcal{D}_{1}^{-\|} \psi_{N N} .
$$

where $\mathcal{N}($ ) is the normallization factor of the affine rooth. Finallity, moving thermis to the lefth-hand side we recover (4.3)) where

$$
L=\left\{\mathcal{N}_{(0)} \sum_{i=1}^{N} \cdots \sum_{k=1}^{N} \mathcal{D}_{N}^{-1} \Lambda_{N i} D_{i}^{-1} \cdots \Lambda_{k 1} \mathcal{D}_{\|}^{-4}\right\}^{-1},
$$

and the spectioall parametter is given by

$$
\mu=-(-)^{\gamma} \lambda
$$

Since each row and columm of $\Lambda$ conttain onlyy one entry the sum will gemeratte a simglle therm, i. e.,

$$
L^{-1}=\left\{\mathcal{N} \mathcal{D}_{N}^{-1} \mathcal{D}_{N-1}^{-1} \cdots \mathcal{D}_{1}^{-1}\right\}^{-1},
$$




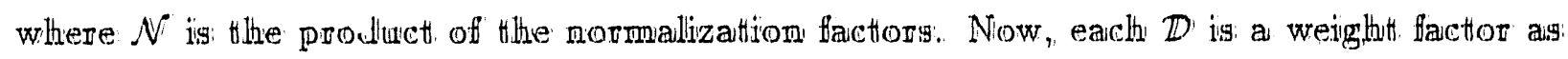
dleffimed im sttep 1. Thrus, there is a dlimect mapping bettween the order of the wreighth factions: amd their locattion in the correspondling weighth dliagram.

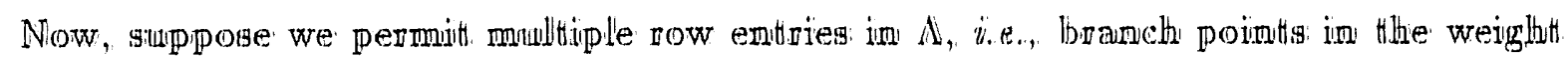
divagram. Firsth consider the casse where such minultiple enttriess occur albove the $N$ the row. As before, there is: a single vacumum statte, and the constrant $\lambda$ is locatted in the wppen righththand conner of $\Lambda$. Therefore, the constrainit $i<k$, for $k \neq 1$, remains in effect for eq. (44.4)).

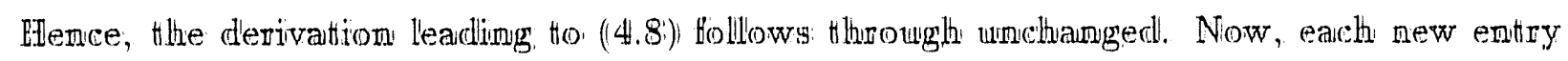

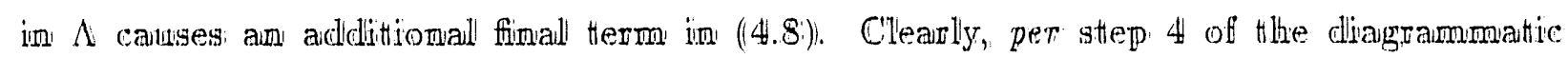
algorithm, the finall $\mathbb{L}$ ax operattor is obtained by thalking the reciprocall of the sum of therms generatied by (4.8)).

Next, suppose the multiple row entries: in $\Lambda$, ditue to the branch poimh, occur in the

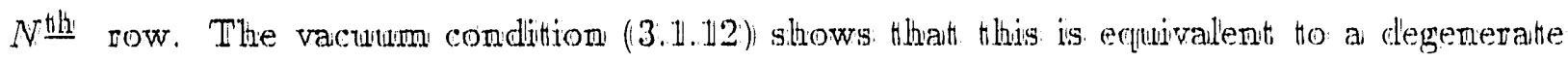
vacuram statte with, say, degeneracy $d$. Silbsequentily, this row will be associanterll witth the eliminatted root, and the $d$ imtegers will be assigmed the value $\lambda$. In fact, $\lambda$ appears only in this row. Clearly, in the weighth diagram the $d$ weighths sthare the same level.

Let us furst disctuss the case where the coefficients $\lambda$ occur in the first row. Thus, Eq.(4.4) remains valid, keeping intact the constraint $i<k$, for $k \neq 1$. Furthermore, the scallar eigenfunction $\phi(x)$ is mow a linear combination of the components $\psi_{N}, \psi_{N}-1, \ldots, \psi_{N-d+1}$, and the equation for $\psi_{1}$ be:omes

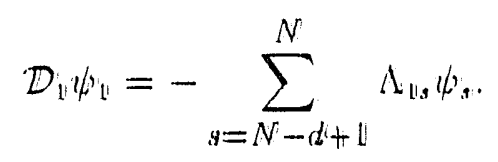

C'onsequently, eq. (14.7) is modiffied tio

$$
\begin{aligned}
\psi_{s}=-(-) \sum_{i=1}^{N} \ldots \sum_{k=1}^{N} \sum_{j=N-d+1}^{N} \mathcal{D}_{s}^{-1} \Lambda_{s i} \mathcal{D}_{i}^{-1} \ldots \\
\times \mathcal{D}_{k}^{-1} \Lambda_{k 1} \mathcal{D}_{i}^{-1} \Lambda_{k j} \psi_{j},
\end{aligned}
$$


where $\beta$ ' is: the number of field replacememts: performed. The chanacteristic equation is

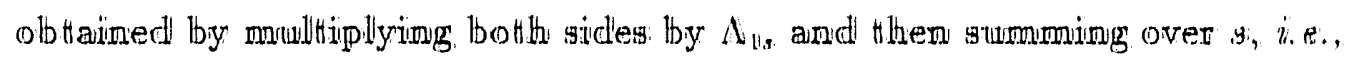

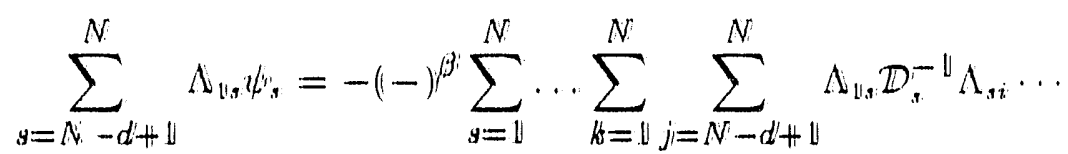

$$
\begin{aligned}
& \times \mathcal{D}_{k}^{-1} \Lambda_{k \nu} \mathcal{D}_{i}^{-1} \Lambda_{l j} i \psi_{j} .
\end{aligned}
$$

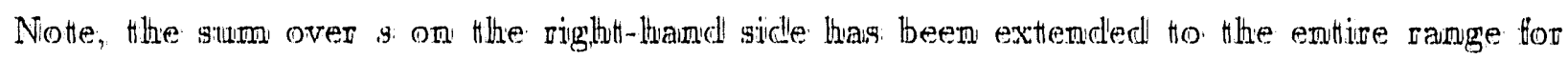
comveniemce.

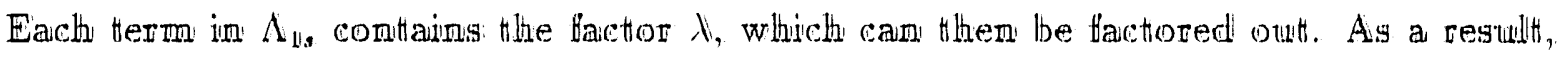
the scallar eigentumetion is foumd to be

$$
\phi=\sum_{s=N-d+1}^{N} \Lambda_{1 ! s} \psi
$$

and the Lax operattor,

$$
L=\lambda\left\{\sum_{s=1}^{N} \ldots \sum_{k=1}^{N} \Lambda_{1, s} \mathcal{D}^{-\|} \Lambda_{s i} \cdots \Lambda_{k, 1} \mathcal{D}_{1}^{-1}\right\}^{-1}
$$

Clearly, this has the same interpretation as the non-degeneratte brancthimg case.

For the last stage of the proof, we relax the condition than multiple occurrences of $\lambda$ must all the in the first row of $A$. In the weighth diagram this means not all the arrows associated with the elliminated root point to the bottiom level. Recall trom the disctussion surrounding eq.(3.1.11), the elimimated root with coefficient $\lambda$ actis as a stathe raising operator. Thus, every occurrence of $\lambda$ will appear in the upper triangular portion of $\Lambda$, and the unit coefficients of the sthatte lowering rootss are in the lower triangular portion.

For $\lambda$ in the $k$ the row of $\Lambda, k: \neq 1$, Eq. (4.4) is modiffied to

$$
\mathcal{D}_{j} \psi_{j}=-\mathcal{N}_{0} x \psi_{k}-\sum_{n \neq k}^{N} \Delta_{j n}{ }_{j n} \psi_{n},
$$

where, aince $\lambda$ correspondts to the sthatle raising operatior, $k>j$. Consider the case where $j$ is: the largest stuch index: to satisfyy this equation. Then, allowing degenerathe vartum 
sttattess, we thave

$$
\begin{aligned}
& \psi_{s}=-(-)^{\beta} \sum_{i=\|}^{N} \cdots \sum_{j=U}^{N} \mathcal{D}_{s}^{-1} \Lambda_{s i} \mathcal{D}_{i}^{-1} \cdots \Lambda_{k j}, \psi_{j}
\end{aligned}
$$

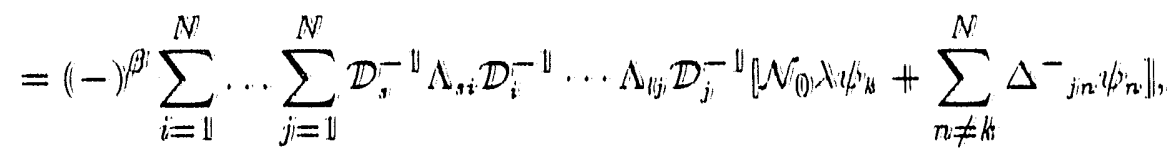

where, again, $\beta$ is the number of field replacements performed. "The effect of the fartor in

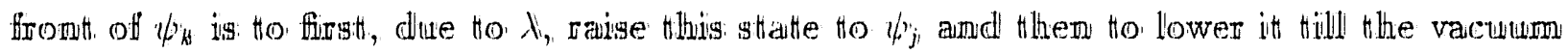
stratte $\psi$ ", is reached. Now, since the correspondling weighth diangram is cyclic, there musth existt some factlor that will circullatte $\psi \psi^{\prime}$, back to $\psi$ '

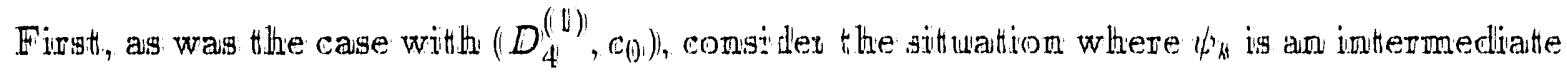
sittatte in $(14.17)$, i.e.,

$$
\psi_{s}=(i-)^{\alpha} \sum_{i=1}^{i V} \ldots \sum_{k=1}^{N} \mathcal{D}_{s}^{-\|} \Lambda_{s i} \mathcal{D}_{i}^{-\|} \cdots \Lambda_{j, k i} \psi \psi_{k t} .
$$

This gives

$$
\psi_{k}=(-)^{\alpha x}\left\{\sum_{i=1}^{N} \ldots \sum_{k=1}^{N} \mathcal{D}_{s}^{-\|} \Lambda_{s i} \mathcal{D}_{i}^{-1} \cdots \Lambda_{j, k}\right\}-\| \psi_{s} .
$$

Thus, per sttep 3 of the diagramuatlic allgorithm, the factior $\mathcal{D}_{k}$, asssociated with the weighth vector att the taill end of both connecting arrows dioes not appear. Further, proceerling from highther weighths to lower weighths in the weight diagram contributhes farthors of $\mathcal{D}_{i}^{-1}$ in the operator defined in step 2 for the linear stulbeliagrarm.

Finally, if $\psi_{\text {, }}$ does not appear as an intermediatte statte of the varum statte $\psi$, in $(4.17)$, then it must occur as an inttermedliatte sthatte for one of the other varum stattes. Again, since the weighth diagram is cyclic, there is some closed patth going from tw, to each of these other vacua. However, to writte down a finall expression is: too unwieldling: Nevertheless, it should be clear thath the general rulles of the diangrammattic allgorithm are complette and provide an accuratte mapping between weight dliagrams and the scallar Lax equation. 


\section{SUPERSYMMETRIC LAX OPERATORS}

In this: section we generallize the diagrammattic scheme to supersymmetric affune Lie algebrass. The classifficattion of all possible simpersymmetric extlemsions of the bassic Lie ail-

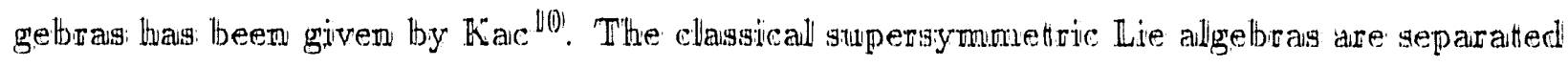
inuto several secies. The umittary series is demoted . $4(m, m)$ or $S L(m+1 \| m+1)$. Next, the ortthosymplectic series $O S p\left(m, \| 2 m_{i}\right)$ includes $B\left(m, m_{i}\right)$ or $O S p\left(2 m+1 \| 2 m_{i}\right), C^{\prime}(m+1)$ or $O S p\left(2 \| 2 m_{i}\right)$, and $D\left(m_{i}, m_{i}\right)$ or $O S p\left(2 m_{i} \| 2 n_{i}\right)$. These basic series are allso called contragradient which means they thave the same number of posittive and negative roots. The exceptionall conttragradient extlensions: are given by $F(4), G(3)$, and $D(2 \| 1 ; \alpha)$ which is a dleformaltion of $D(2,1)$ and is the only Lie allgebra dependent on a continutus parametter. Finallly, there are the non-comtrangradienti or strange cases $P(n)$ arvd $Q\left(n_{i}\right)$.

In addition to the boronic simple rootis of the basic Lie algebra, the aimple root systhem of the supersymmettric algebras contains two distimet kindls of fermionic roots. The Dynkin symbol! of the first type is sometimes given by a shaded vertex representing a non-zero norm. The second fermionic root type has zero norm whose Dynkin symbol is given correspondlingly by a crossed out vertex. As always, the bosonic root is denotherl by a white vertex.

A new feature occurring in the supersymmetric Lie algebras is that they may have severall non-equivalent simple root systems, corresponding to different Dynlkin diagrams and Cartian mattrices. In other words, the different root systems can not be transtiormed into each other through standard Weyl rotations. Insthead, they are obtained by performing the "Weyl" transtormattion with respect to the nilpotent fermionic root. For more detaills,

see Erappatt et al. It which also presents a large collection of Dynkin cliagrams aissocianted with alli of the classical contragradlient supersymmetric cases, thothe of the affine and tiwistled 
affine supersymmetric allgebram.

Non-equivallent simple root systems which represent the same supersymetric Lie algebva differ in the distribution of bosonic and fermionic roots. However, there we are intterested in considlering a wathurall extension of the Drinffeld-Sokolov proced hure to the supersymmetric case. This restricts our choice of the simple root system for build ding the supersymmetric Lax operattors ${ }^{12}$. Recall, in the bosonic case the mRdV Lax operattor constructed with the grardation choice of Drimfeld and Sololow generattes Torla lattice models ${ }^{1,13,144}$. For stupersymetric algebras it has been shown ${ }^{45}$ that Toda lattices are possitble only for simple root systems composed purely of fermionic roots. This choice leards to commuttation relations

$$
\begin{array}{cc}
{\left[H_{i}, H_{j}\right]=0,} & \left\{E_{i}, F_{j}\right\}=\delta_{i j} H_{i}, \\
{\left[H_{i}, E_{j}\right]=A_{i j} E_{j},} & {\left[H_{i}, F_{j} \|=-A_{i, j} F_{j},\right.}
\end{array}
$$

where $0 \leq i, j \leq r$. The Cartian matrices can be obtained from the Dynkin diagram as in the nom-supersymmetric case, althougth due to the zero-norm fermionic roots we now write

$$
A_{i j}=\left(F_{i}, F_{j}\right)
$$

Supersymmetric Lie algebras with purely fermionic root systems thave been given by Leittes et al. 16 ;

$$
S L\left(n+\mathbb{\|} \| n_{i}\right), O S p\left(m_{i} \| 2 n_{i}\right):\left(m_{i}=2 n, 2 m+2,2 n_{i} \pm 1\right), D(2 \| 1 ; \alpha) .
$$

Further, the infinite-dimensional affine supersymmetric Lie algebras with purely termionic simple root sysitems are

$$
S L(n \| n)^{(1))}, O S p(2 n+2 \| 2 n)^{(1)}, D(2 \| 1 ; \alpha)^{(1)},
$$

while the infinite-dimensionall twisted affine cases are

$$
\left.S Q(2 n+1))^{(2)}, S L(n \| n)\right)^{(2)}, \operatorname{OSp}\left(2 n_{i} \| 2 n_{i}\right)^{(2) !} .
$$


The supensymmetric extension of the $K d V$ equations was finst discussed in Mlanin and Radull ${ }^{17}$. They suggested replacing the bosonic denivative $\partial_{x}$ by its supersymmetric analog, i.e.,

$$
\partial_{x} \rightarrow D=\frac{\partial}{\partial \theta}+\theta \cdot \frac{\partial}{\partial x}
$$

Note that $D^{2}=\frac{\partial}{\partial x}$. The system of matrix equations of Drinfeldi and Sololov can then be generalized to ${ }^{12}$

$$
\mathcal{L} \vec{\psi}(x, \theta) \equiv[D+Q(x, \theta)+\Lambda] \vec{\psi}(x, \theta)=0
$$

where $\Lambda$ is generated by the punely negative fenmionic roots, and $Q(n, \theta)$ is a Grassmann odd fermionic supenfield which can be expanded as

$$
Q(x, \theta)=\sum_{i=1}^{r} H_{i} \Psi_{i}(x, \theta),
$$

where now $H_{i}$ are elements of the Cartan-Kac subalgebra. The vacuum condition is as before,

$$
\Delta^{-} \vec{\psi}(x, \theta)=0
$$

Since the second type of fermionic root is nilpotent, they deserve special trentment when constructing cyclic weight diagnams. To illustrate how this comes about, consider the fundamental representation of the supersymmetric algebra $\operatorname{OSp}(2 \mid 2)$. The Dynkin diagram of the purely fermionic root system is given by<smiles>C[Se][O]</smiles>

where both fermionic roots are denoted as having zero norm, and where we have indicated the choice (11) for a highest weight vector. The Cartan matrix is then easily found to be

$$
A=\left(\begin{array}{cc}
0 & -2 \\
-2 & 0
\end{array}\right)
$$

To construct the highest weight diagram we proceed as before. Since a positive one appears

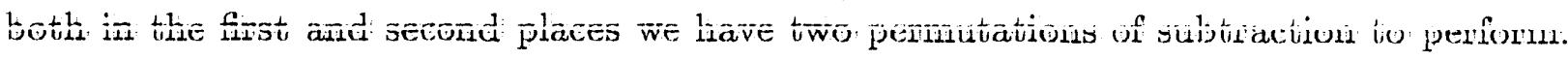


In panticular, we can stant by subtracting the finst now of the Cartan matrix giving (113), and then subtracting the second now resulting in (33). However, unlilke the bosonic case, we may not subtract the first now of the Cantan another time from the weight (13). This is because here the fermionic weight vectors are nilpotent and subtracting any Cartan row twice gives a decoupled state. Similarly, we can start by sulbtracting the second Cartan row once(and only once) and then the finst row giving (33). Thus, we find the weight diagram with height two:

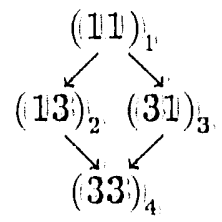

The decoupling which occurs when constructing a cyclic weight diagram for an affine supersymmetric algebra is almost as straightforward. For example, in figure 6 we display the partially decoupled weight diagram of $\left(S L(2 \mid 2)(1), c_{0}\right)$ where states were decoupled as we went from top to bottom. There are several ways to decouple the remaining weights since the lowering operators $b_{0}, b_{2}$ and $b_{3}$, still appear more than once. The only way for a cyclic weight diagram to emerge is by decoupling the weights outside the box. To see that this is also consistent, note that all paths leading from weight 5 to weights 2 or 4 require two applications of $b_{0}$.

To construct a super-Lax operator let us take the vacuum solution $\phi=\Psi_{4}$. We easily find the super-Lax operator to be

$$
L=\left[D+\Psi_{3}\right]\left[D+\Psi_{2}+\Psi_{3}\right]\left[D+\Psi_{1}+\Psi_{2}\right]\left[D+\Psi_{1}\right]
$$

As in the non-supersymmetric case, Lax operators corresponding to the other three vacua are obtained thrugh cyclic permutations of the above operatior. 


\section{DISCUSSTONIS}

In this paper we have shown how one can read off from cyclic weighth diagrams, as. sociated with representations of affine Lie algebras, Lax operators in the diagnnal gauge. This method is most useful when tables of matrix representations are not at hand and must be generated by weight diagnams anyway. Furthermore, this procedhure can easily be implemented on computer by virtue of the fact that computer generated allgorithms currently exist for building highest weight diagrams ${ }^{18}$. With minor modifications, these programs can be adapted for cyclic weight diagrams.

It remains to be seen whether higher representations lead to any new physics. If so then a program of categorizing these results might be pursued to identify redind ant solitions. This might be easier to answer for supersymmetric algebras since nilpotency projects out decoupled weight vectors. What is clear though, at least for the non-supersymmetric: cases is that these higher representations lead to integrable systems. Recall, to prove the integrability of $\mathrm{KdV}$ systems Drinfeld and Sokolov found the necessary infinite set of conserved currents to be given by the coefficients of the Laurent expansion of $L$ in the affine parameter $\lambda$. Our conclusion follows from the fact that every representation of a basic Lie algebra has an affine extension, and that defining properties of affine Lie algebras are representation independent.

Finally, it would be interesting to see if our procedure could be modified to directly generate Lax operators in other gauges. Furthermore, in light of recent work ${ }^{9}$ on generalizations of the Drinfeld and Sokolov scheme, one may also consider diffenent gradations of the affine Lie algebra from which to obtain the matrix $\Lambda$ and the form of $q(x)$. 


\section{ACKNIOWLEDGEMENTS}

We wish to acknowledge Zongan Qinu and Moustafia Awadla for discussions, and Pienre Ramond for reading over the manuscript.

\section{R:EFERIENCES}

1. V. Drimfeld and V. Sokolov, J. Sov. Math. 30, 1975 (1984)!

2. V. A. Fateev and S. L. Lulk'yanov, Imt. J. Mlod. Phys. A3, 50 (11988):

3. S. L. Carbon and E. J. Piand, UF preprint, IFT HEP GIL-111 (1991):

4. An excellient review of Lie algebra techniques is R. Slansky, Phyrs. Rep: 79,1 (1981).

5. E. B. Dynkin, Amer. Math. Soc. Trans. Ser, 2,6, 1111 and 246, (195, $)$.

6. Highest weight talbles corresponding to many repnesentations of simple Lie algebras can be found in M. R. Bremmen, R. V. Mloody and J. Patera, Tublies of Dominant Weighti Multiplicities for Representations of Simple Lie Alligelinay, (Delkker, New York, 1985):

7. A review of the construction of affine Lie algebras is given in P. Goddard and D. (Dlive, Int. J. Mod. Phys. A1, 303 (1986). Anotiher standard refenence is V. G. Kac, Infinitie Dimenaional Lie Algebmas, (Cambridge university press, Cambridge, 199(0))

8. A. Das, Intiegrable Modelis, (World Scientific,Singapore,11989).

9. Ml. F. De Groot, T. J. Hollowood and J. L. Miramontes, Primceton preprint PUPT-1251 (1991).

10. V. G. Kac, Adv. in Matti. 26, 8. (19777).

11. R. Frappat, A. Sciarrino and P. Sorba, Comm. Math. Phys. 121, 457 (1989)

12. T. Inami and H. Kanno, Comm. Math. Phys. 136, 519) (1991),

13. A. Bilal and J.-L. Gervais, Physs. Lett. B206 412 (1988); Nucl. Phys. B314, 646 (1989)!.

14. P. Forgács, A. Wipf, J. Balog, L. Fehér and L. O'Raifeartiaigh, Phys. Lett. B227, 214 
(1989)).; J. Ballog, L. Felhér, L. O'Raifeartiaighth, P. Fongács and A. Wipt, Anm. Plhys. 203, $76 ;(19990)$

15. M. A. Ollchanetisky, Comm. Matth. Phys. 88, 63: (1983).

165. ID. A. Leithes, Mc. V. Saveliev and V. V. Serganova, Serpulchov preprint, IHEP 8.5-81 (1985).

17. Yui. I. Manin and A. O: Radhall, Comm. Mlath. Plhys., 98, 65, (19855).

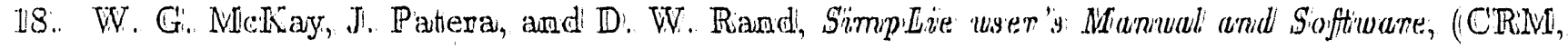
Montreal, 1990i): 


\section{FTGURIE CAPTIONS}

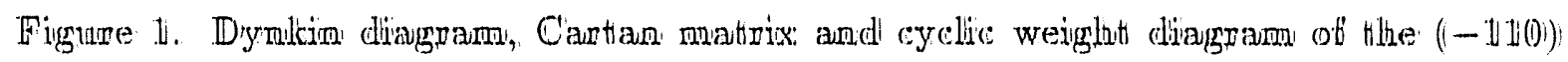
representiation of $A_{2}^{((l))}$ ?. "The dasthed arrow is the dleletted pooth.

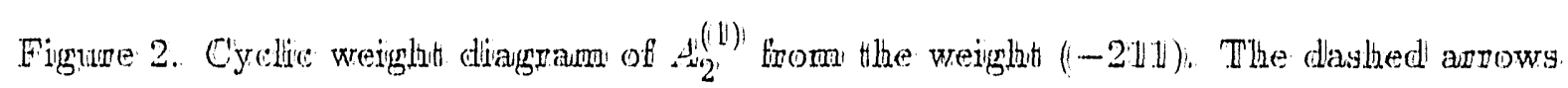
are the deleted rocti.

Figgure 3: Highthest. weighth diagram of the ardjoint represtentaation of $A_{2}$, (111).

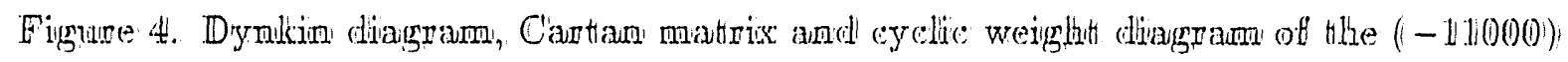
representiation of $D_{4 !}^{(1) !}$. The diasthed arnows are the deleted rooti.

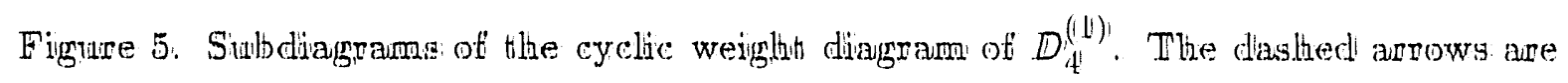
the deleted root.

Figure 6. Dyndkin diagram, Cartian matrix: and complete weighth diangram of the (100) repressentation of $S E(2 \| 2)(11)$. The dhashed arrowrs are the deletted root. The bow enclloses. the cyclice weightht diagram, exchudting the decoupled weighthtis otuthicile. 

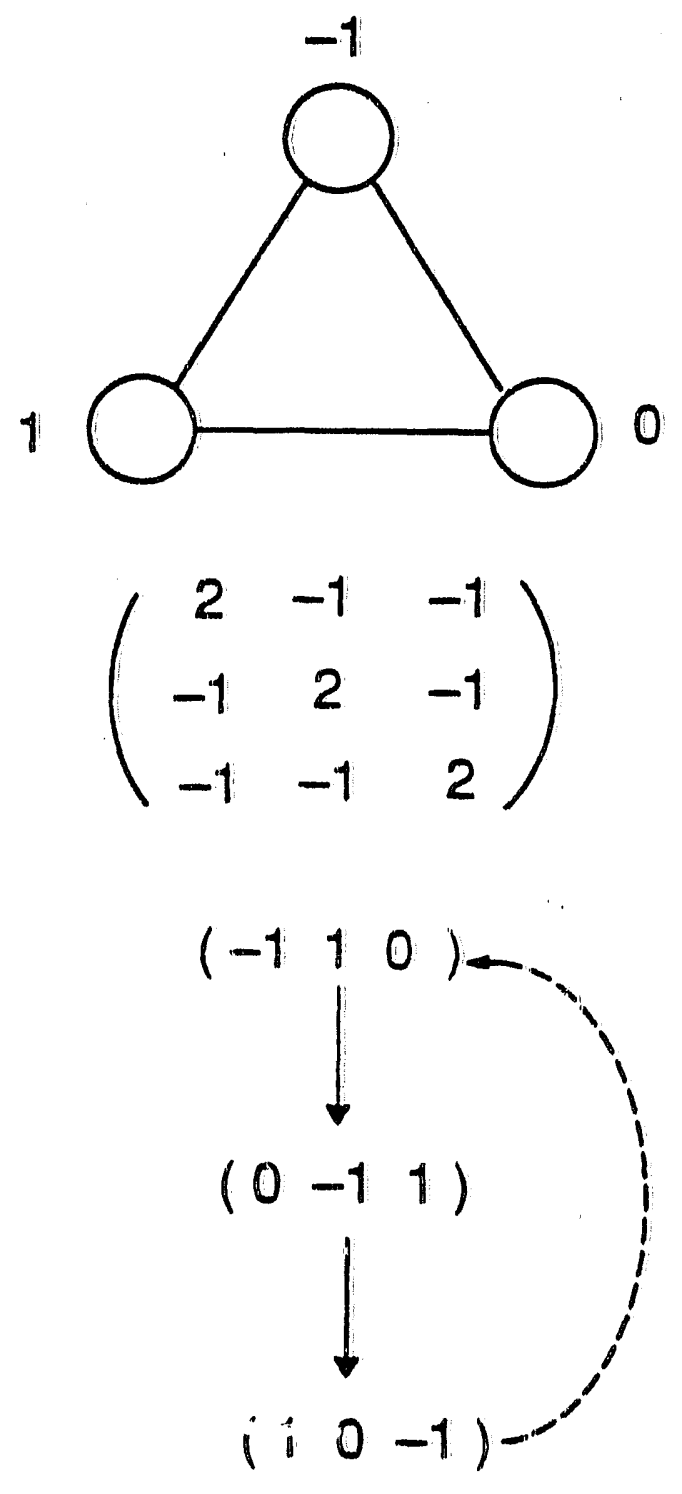

Figure 1 


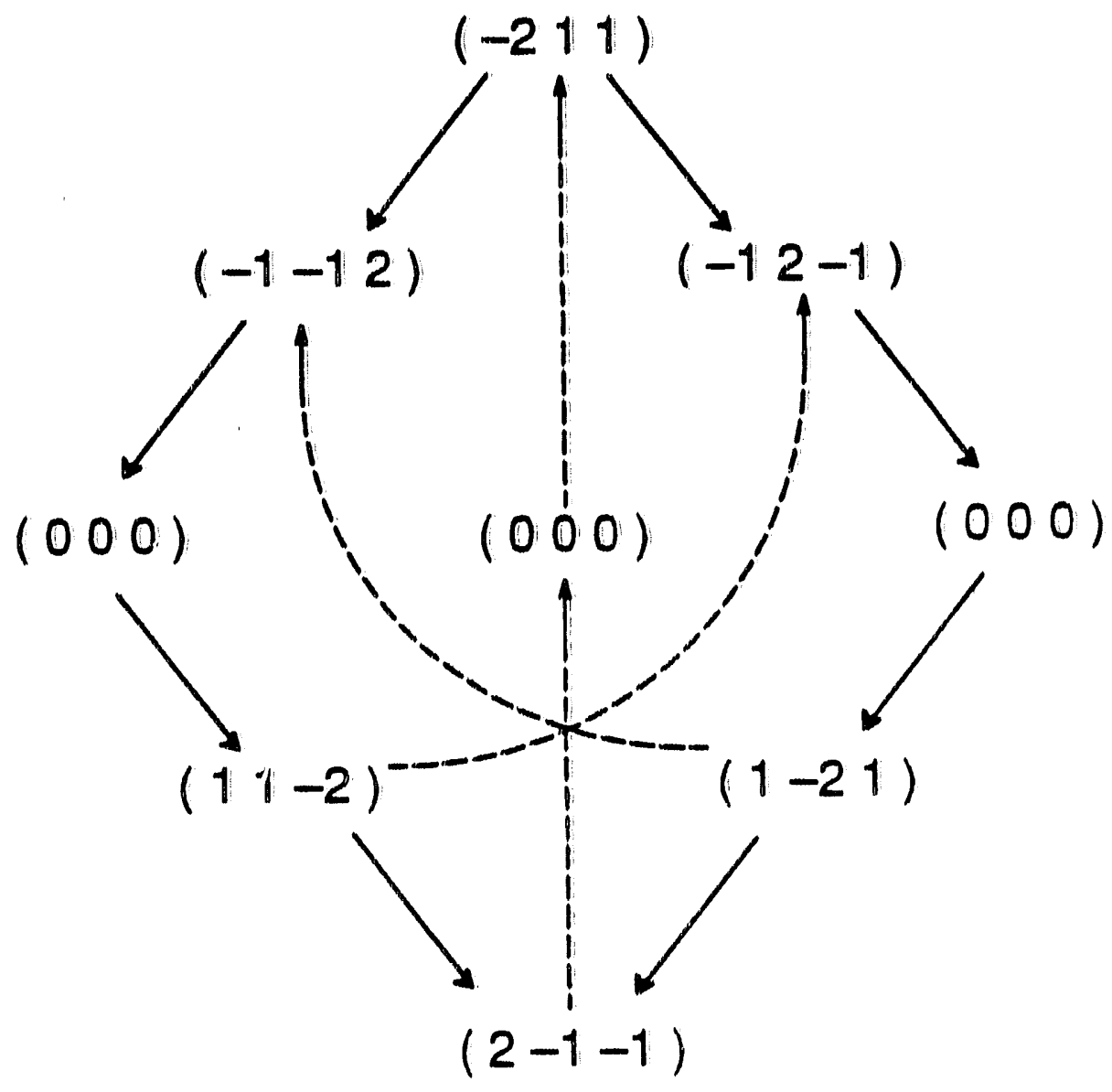

Figure 2 


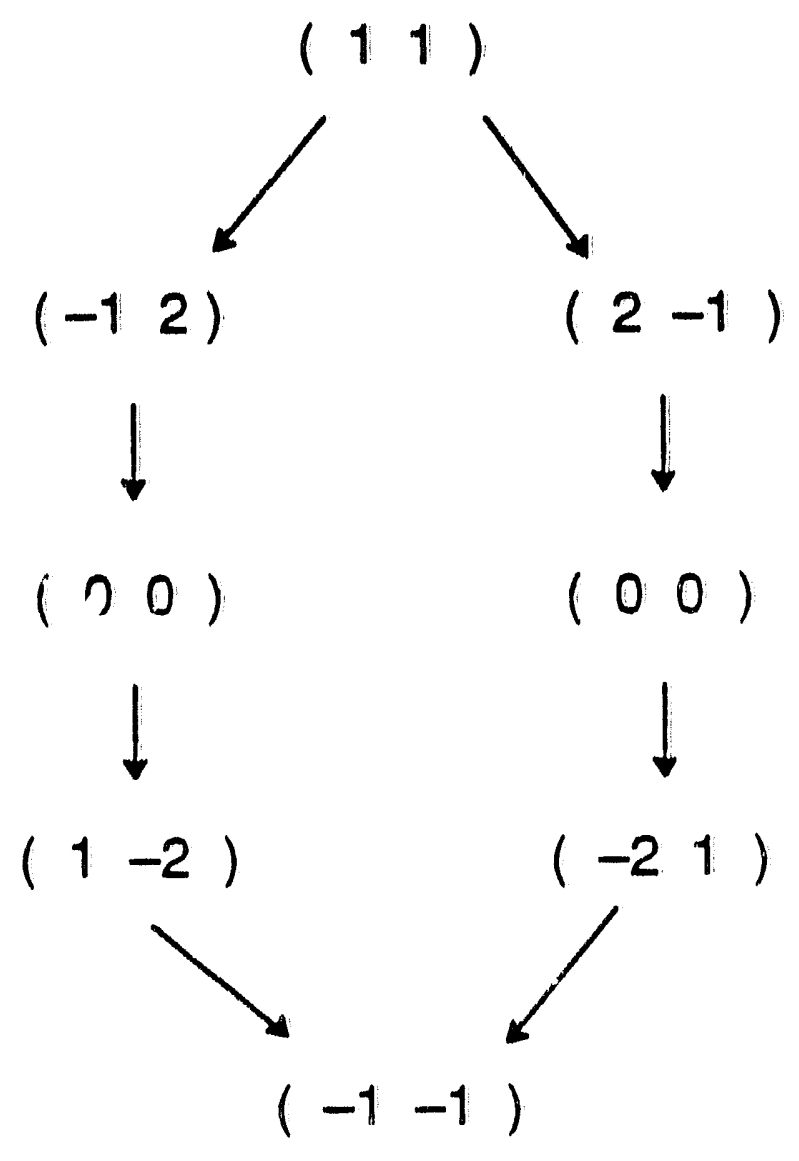

Figure 3 


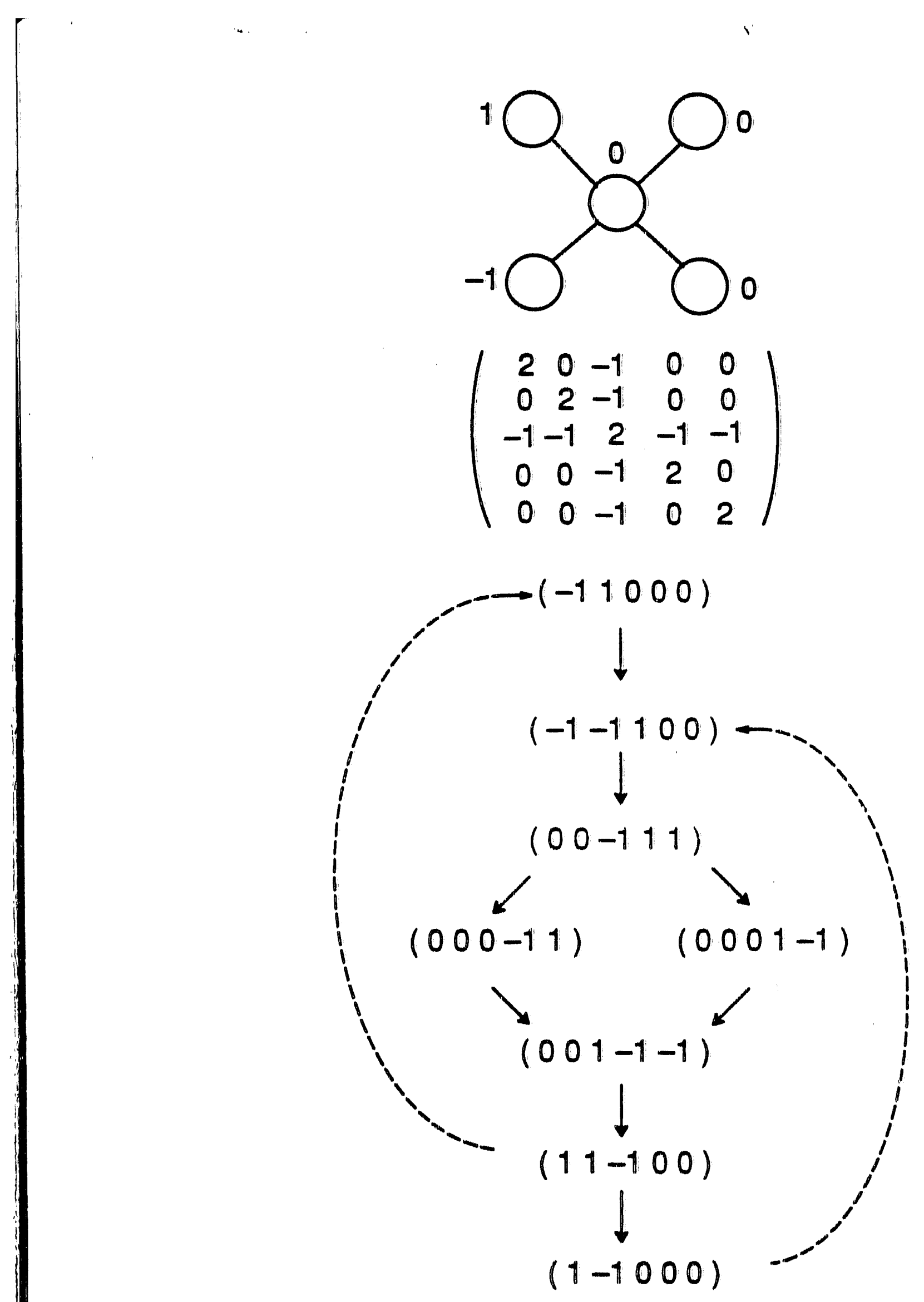

Figure 4 

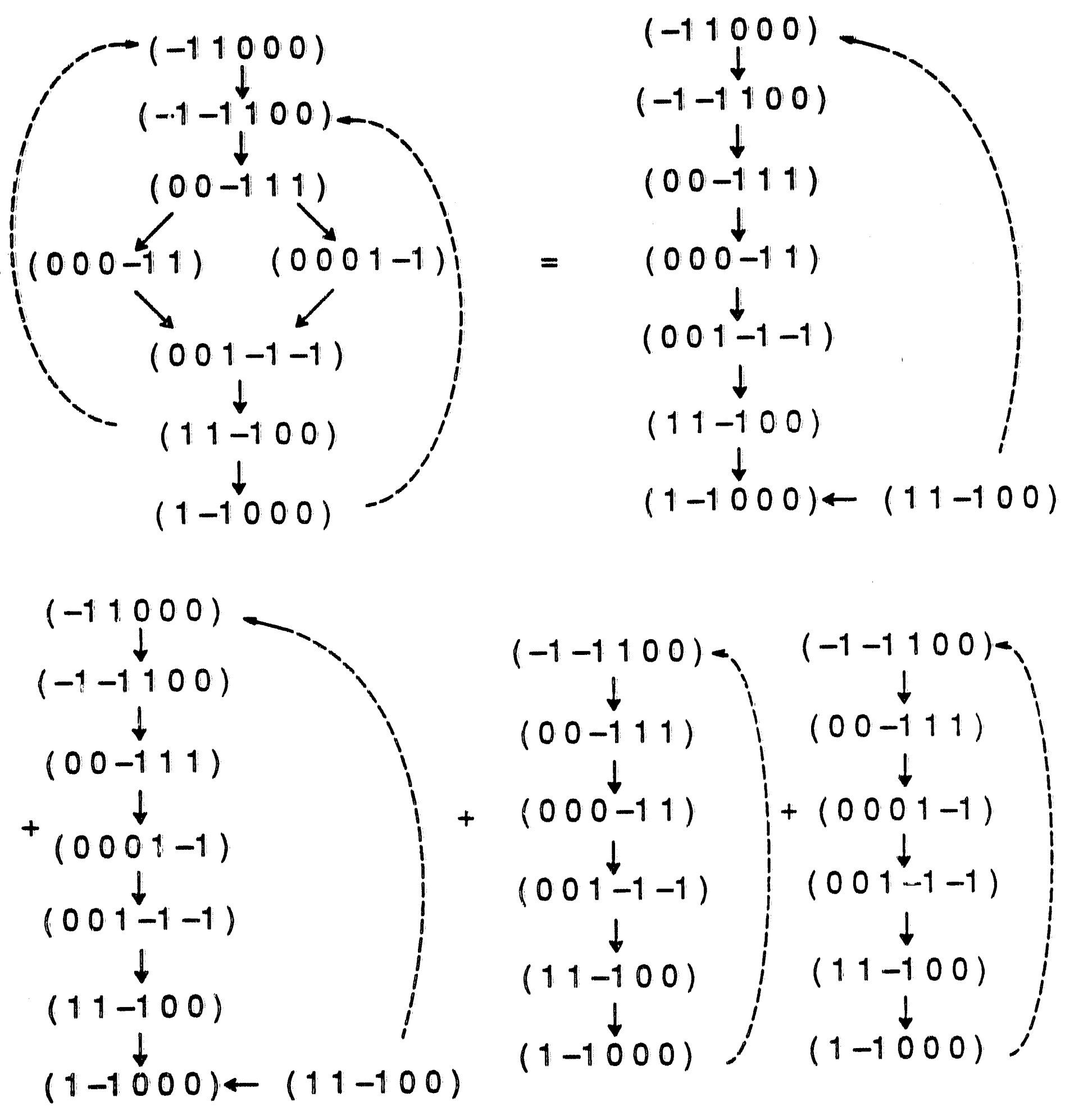

Figure 5 


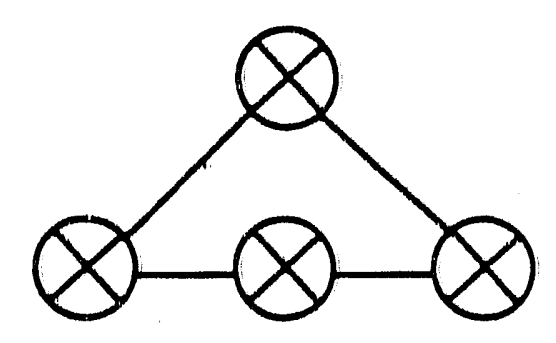

$$
\left(\begin{array}{cccc}
0 & 1 & 0 & -1 \\
-1 & 0 & 1 & 0 \\
0 & -1 & 0 & 1 \\
1 & 0 & -1 & 0
\end{array}\right)
$$

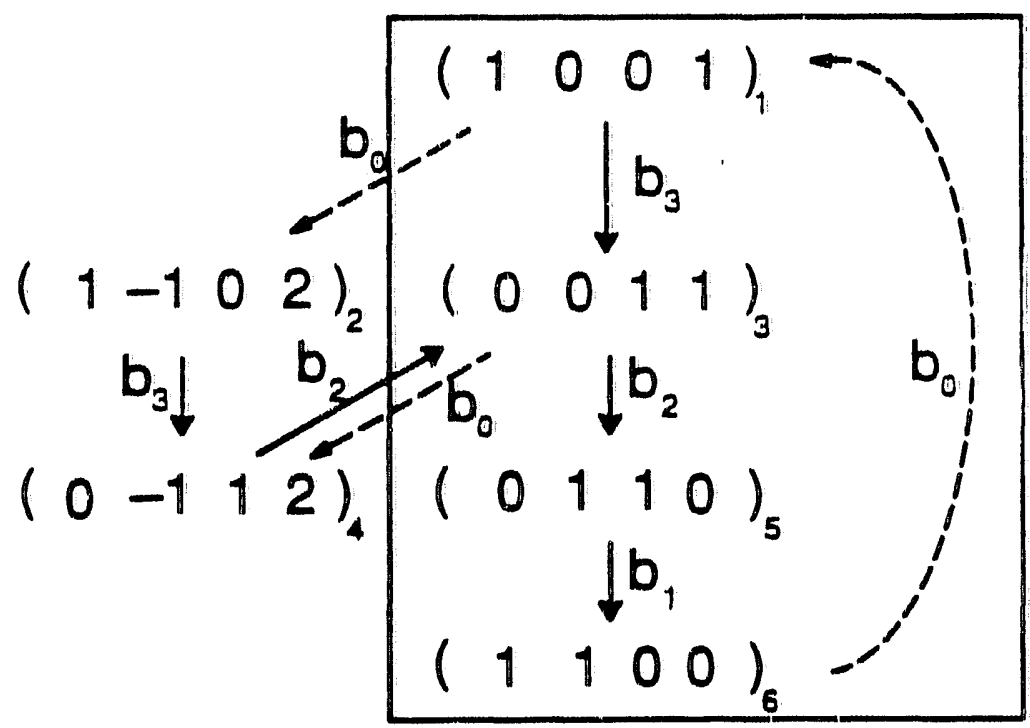

Figure 6 

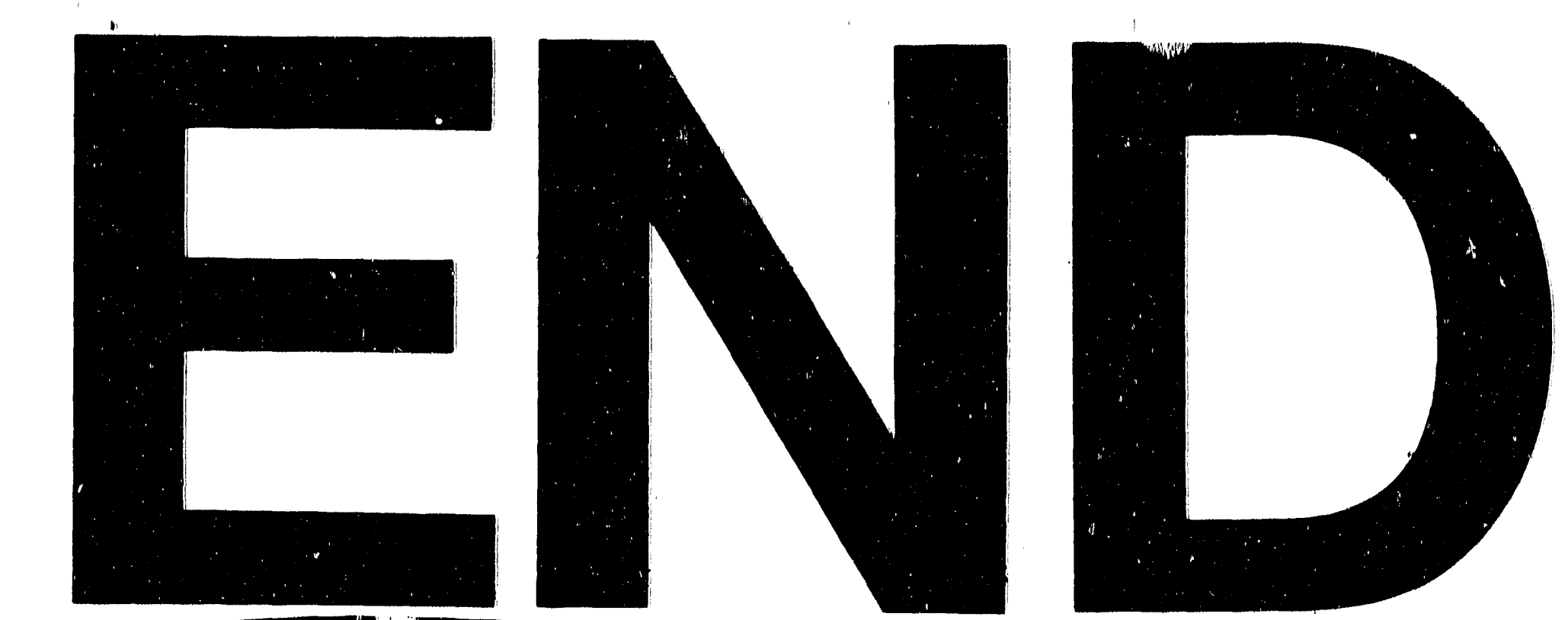

4
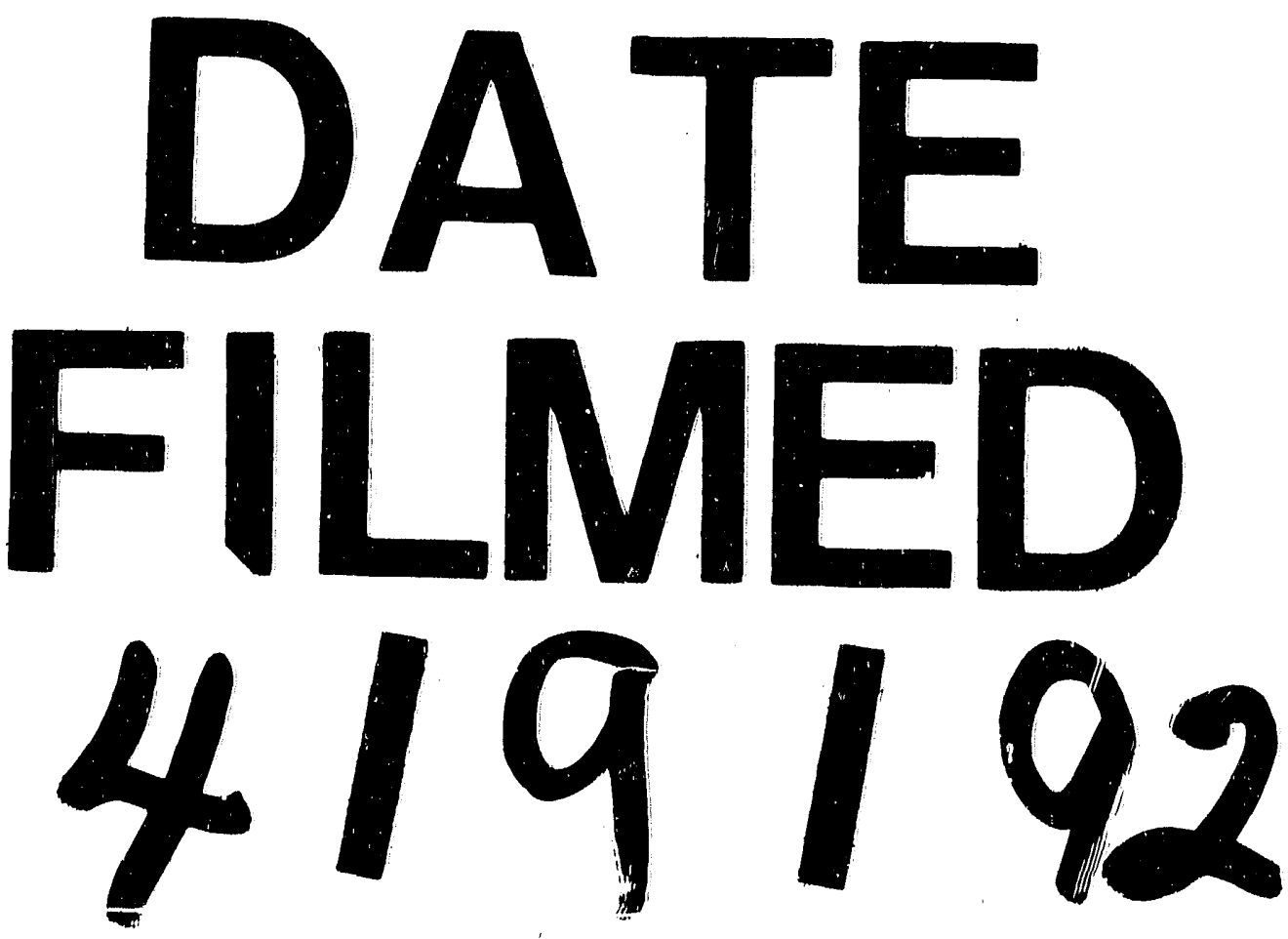

$I$ 


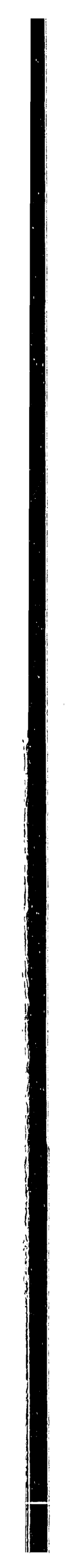

